\title{
Case Report: An Unusual Osseous Lesion
}

\author{
Benjamin Riviere ${ }^{1}$, Thérèse Rousset ${ }^{1}$, Luc Bauchet ${ }^{1,2}$, Nicolas Menjot de Champfleur ${ }^{3}$, \\ Vanessa Szablewski ${ }^{1}$, Valérie Costes ${ }^{1}$, Valérie Rigau ${ }^{1}$ \\ ${ }^{1}$ Department of Histopathology, Saint Eloi-Gui de Chauliac Hospital, University Medical Center, Montpellier, France; ${ }^{2}$ Department \\ of Neurosurgery and INSERM U1051, Saint Eloi-Gui de Chauliac Hospital, University Medical Center, Montpellier, France; \\ ${ }^{3}$ Department of Radiology, Saint Eloi-Gui de Chauliac Hospital, University Medical Center, Montpellier, France. \\ Email: v-rigau@chu-montpellier.fr
}

Received March 18 ${ }^{\text {th }}, 2013$; revised April 18 ${ }^{\text {th }}, 2013$; accepted April 28 ${ }^{\text {th }}, 2013$

Copyright (C) 2013 Benjamin Riviere et al. This is an open access article distributed under the Creative Commons Attribution License, which permits unrestricted use, distribution, and reproduction in any medium, provided the original work is properly cited.

\begin{abstract}
The intraosseous benign notochordal cell tumor (BNCT) is an intravertebral lesion derived from notochordal tissue. The notochord develops in humans during the third week of embryonic life and persists in adults as the nucleus pulposus of the vertebral disks. The most common locations of such tumors are the saccrococcygeal region and the skull base. Most tumors are asymptomatic and small. Magnetic resonance imaging is useful in their detection and precise localization. Histologically, these lesions consist of sheets of adipocyte-like vacuolated or less vacuolated eosinophilic tumor cells with eccentrically located round nuclei without myxoid matrix. The tumor cells express the epithelial markers, vimentin and S100 protein. Main differential diagnosis is chordoma. Intraosseous BNCT do not require any surgical management and should be recognized by pathologists to prevent unnecessary radical surgery. These lesions should be followed-up with conventional MRI.
\end{abstract}

Keywords: Intraosseous Benign Notochordal Cell Tumor; Osseous Tumor; Histopathology

\section{Case Report}

We present a fifty-two year old woman with a personal medical history of fibromyalgia and breast cancer and family history of colorectal cancer. A random medical check up revealed the presence of a monoclonal gammopathy suggesting an underlying immunoproliferative disorder. Subsequent radiological workup demonstrated an anomaly of the third sacral vertebrae. Magnetic resonance imaging of the spine showed a $2 \mathrm{~cm}$ intraosseous lesion hypointense on T1-weighted imaging, hyperintense on T2-weighted images, enhanced after contrast infusion and slightly hyperdense on non enhanced CT scan (Figure 1(a)). There was no expansive soft tissue mass nor osteolysis.

A surgical biopsy was taken with a clinical diagnosis of possible myeloma.

\section{Pathological Findings}

A histological assessment of the specimen revealed normal trabecular bone. The intertrabecular marrow was dominated by the proliferation of large clear cells (Figure 1(g)). These adipocyte-like cells had clear vacuo- lated cytoplasm and eccentrically or centrally located round nuclei (Figure 1(h)). No mitotic figures, anisokaryosis or cytonuclear atypia were recognized. Alcian Blue and PAS staining did not demonstrate intracytoplasmic glycogen granules. There was no myxoid matrix. Immunohistochemistry showed positive staining of the cells for vimentin, S100 protein, EMA (epithelial membrane antigen) and cytokeratin AE1/AE3 (Figures 1(i) and (j)). KI 67 (MIB-1) was $<1 \%$ and CD10 was negative. The radiological and pathological findings suggested a diagnosis of intraosseous benign notochordal cell tumor. The monoclonal gammopathy in the blood was finally considered to be an incidental observation and had no later implications.

\section{Follow Up and Course}

Based on these pathological and radiological observations, simple clinical and radiological follow-up was proposed. Four years later the lesion was radiologically unchanged (Figures 1(b) to (f)). The patient is presently followed annually by lumbosacral magnetic resonance (MR) images, computed tomography (CT) scans, and osseous scintigraphy. 


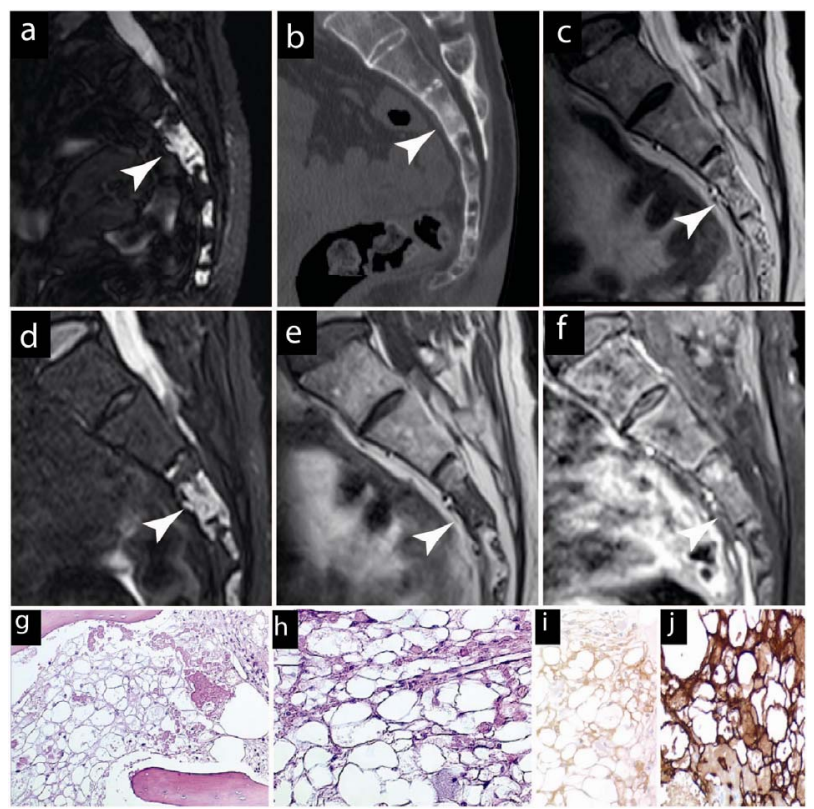

Figure 1. MRI of the spine (a) showed signal abnormalities at the level of S3 (white arrowhead), S4, S5, Co1 and Co2 on STIR sequences. Four years ((b) to (f)) follow-up showed no extent of this signal abnormality, appearing hyperdense on non-enhanced CT scan (b), hyperintense on T2-weighted images (c), hyperintense on STIR sequence (d), hypointense on T1-weighted images (e), with slight enhancement after contrast infusion (f). Replacement of the inter-trabecular marrow by a proliferation of clear and large cells (HES $\times 200$ ) (g). Physaliphorous cells: proliferation of adipocyte-like vacuolated cells with eccentrically small nuclei without anisocaryosis (HES $\times$ 400) $($ h). Immunohistochemistry: strong cellular reactivity for S100 protein (i) and cytokeratin AE1/ AE3 (IHC × 400) (j).

\section{Discussion}

The notochord is developed in humans during the third week of embryonic life and has a role in the formation of the vertebral column and the neural plate [1]. During subsequent development of the embryo, the notochord regresses in the region of the vertebral bodies and persists as the nucleus pulposus of the intervertebral disks $[1,2]$. Notochordal remnants can be found in the intervertebral disks, as "rests" or "vestiges", and disappear by the age of 1 to 3 years [3]. Ectopic notochordal rests can occur along the midline of the craniospinal axis, from the dorsum sella to the coccyx, mainly at the base of the skull [4].

About 160 years ago, these lesions were first described by Luschka and named ecchondrosis/ecchordosis physaliphora spheno-occipitalis [5,6]. Later Muller and Ribbert found that the lesion derived from notochordal tissue [7]. Intravertebral notochordal remnants were first described in 1982 and called ecchordosis physaliphora vertebralis. These observation were made at autopsy [1]. Since then, a number of such microscopic remnants have been found incidentally at autopsy, generally less than 1 cm $[1,8]$. More recently, larger intravertebral notochordal lesion seen radiologically have been described. In 1996, Darby et al. described the first "giant notochordal rest", comparable to remnants found in the intervertebral disk and ecchordosis physaliphora [1]. It has been argued that these lesions are histologically different from vestiges and notochordal rests [9]. As such, terms like "benign chordoma" or "giant notochordal hamartoma of intraosseous origin" have been suggested [1]. Finally, the term intraosseous benign notochordal cell tumor (BNCT) was recently adopted, including both small lesions found incidentally and larger lesions that have become detectable on radiological images or have been symptomatic $[1,9$, 10].

Benign notochordal cell tumors are intraosseous lesions which can occur along the midline of the craniospinal axis, particularly in the saccrococcygeal or sphenooccipital region $[8,10]$. A recent autopsy series found BNCTs in $20 \%$ of 100 cadavers, with a mean age of 63 years (7 to 82 years), with a distribution of $12 \%$ in saccrococcygeal vertebrae, $11.5 \%$ in the clival region, $5 \%$ in cervical vertebrae, $2 \%$ in lumbar vertebrae and none in the thoracic vertebrae [8].

Benign notochordal cell tumors are usually asymptomatic [10]. When they do give symptoms, the most prominent is back pain $[1,3]$. Other symptoms related to the location of the lesion have been reported (coccydynia, limitation of movement, numbness, pain of fingers, neck, buttock and shoulder stiffness) [1]. Radiologically, BNCTs appears hypointense on T1-weighted and hyperintense on T2-weighted MR images [1,3,10,12]. There is no contrast enhancement after injection of gadolinium [10-12]. On CT scans the lesions are often sclerotic [10,12]. These lesions are well circumscribed. The smaller BNCTs are not seen radiologically and are incidental microscopic findings.

Histologically, BNCT is an unencapsulated lesion filling the intertrabecular spaces and consisting of nests or sheets of clear cells resembling adipose tissue on low magnification, [1,3]. They have clear cytoplasm and eccentrically located round or oval nuclei [1,9]. Smaller granular cells are seen with eosinophilic globules of condensed cytoplasm. These cells show centrally located enlarged nuclei with rare small nucleoli $[1,3,9]$. The physaliphorous cells are "bubbly" cells containing multiple clear cytoplasmic vacuoles [1]. In some cases, cystic spaces are present and contains eosinophilic colloidlike material being positive for Alcian blue and periodic acid-Schiff $[1,9]$. There is no intercellular stroma or myxoid matrix [1,3]. The cell proliferation is relatively uniform [1]. There are no mitoses, atypia or nuclear pleomorphism, no fibrous bands, lobulation or necrosis [1,3,9]. Surrounding bone trabeculae are often sclerotic [3,9]. The lesion can 
harbour some residual islands of normal haematopoietic marrow cells [1].

Immunohistochemically, the tumor cells stain for vimentine, S100 protein and epithelial markers like epithelial marker antigen (EMA) or cytokeratins (AE1AE3) $[1,10]$. They show a low $(<1 \%)$ KI-67 (MIB-1) proliferation index [1]. The same immunoprofile is found in nontumor notochordal tissue and chordomas [1].

The evolution of BNCTs is indolent, with a benign behaviour and appears to be relatively frequent compared to chordomas [1].

BNCTs can be observed with no particular treatment, but do need a long-term follow-up [1]. The follow-up program is based on CT scans and MR images, and should be focused on excluding osteolysis or extension to soft tissue. Such transformations are indicators of chordoma development [3]. If symptomatic, surgery can be an option [1].

The histologic similarity of the notochordal vestiges of intervertebral discs, ecchordosis physaliphora, BNCTs and chordomas are important. Intervertebral vestiges are persisting notochords within the intervertebral disks in adults [1]. The main difference is their location because vestiges are present in the center of the intervertebral disk as opposed to the vertebral body for BNCT [1]. Vestiges are described as a cysticlike space in the annulus fibrosus [1]. Histologically, they are composed of cords or strands of notochordal cells embedded in a myxoid matrix [1]. The presence of this intercellular myxoid matrix differenciates them from BNCTs.

Ecchordosis physaliphora are extraosseous, ectopic notochordal lesions of embryologic origin, similar to notochordal vestiges of the intervertebral disk $[1,5,6]$. They are often located intracranially, on the dorsum of the clivus and in the prepontine cisterna [13]. They can be found at any level from the dorsum sellae to the saccrococcygeal region and are usually present extradurally [13]. Ecchordoses grow slowly and in most cases are clinically asymptomatic [7]. Some rare cases do become symptomatic trough tumour expansion, brain compression or haemorrhage [13]. Because of their location, the differential diagnosis with BNCTs is easy [1]. In contrast to chordoma, ecchordosis physaliphora is small $(<3.5$ $\mathrm{cm}$ ), shows no contrast enhancement on MR images and are similar histologically to intervertebral notochordal rests with the same immunohistochemical profile as the other notochordal lesions $[1,4,13]$.

The main differential diagnosis to BNCT is chordoma, a low grade malignant neoplasm of notochordal origin. Chordomas can occur anywhere in the axial skeleton and common locations are the base of skull and saccrococcygeal vertebrae $[8,10]$. Chordomas are aggressive neoplasms characterized by slow growth with local destruction of bone [1]. These tumors are larger, ranging in size from $50 \mathrm{~mm}$ or more and can involve more than one vertebral body [1]. Symptoms frequently include pain and neurological deficits. Distant metastases have been reported in up to $43 \%$ of patients and the overall median survival time with chordoma has been estimated to be approximately 6 years [1].

Radiologically, chordomas also appear hypointense on T1-weighted and hyperintense on T2-weighted MR images but demonstrate heterogeneous and marked contrast enhancement after injection of gadolinium [3,4,10,12]. There is often extensive lytic bone destruction, presence of an expansive soft tissue mass and intratumoral calcification on CT scans [3,10,12].

Macroscopically, chordomas present as a gelatinous lesion with hemorrhagic areas and bone destruction [1]. Microscopically, a chordoma is composed of irregular strands or cords of atypical chordoid cells in an intercellular myxoid matrix $[1,3,10]$. On low magnification microscopy, the lesion has a lobularity with fibrous septae [1]. The cells can be large with pale cytoplasm and intracytoplasmic septations or abundant eosinophilic cytoplasm $[1,3,10]$. There are atypical hyperchromatic nuclei, necrosis can be seen, and mitotic figures are found $[1,3]$. Destruction of adjacent bone is present [1]. An immunoprofile equivalent to BNCT is found (EMA, protein S100 and vimentine positivity). However, p53 overexpression and increased KI-67 (MIB-1) indicate the increased proliferative activity $[1,10]$. Recent studies have demonstrated that brachyury is a useful marker for the diagnosis of chordomas. Brachyury is a key transcription factor involved in the early specification of the posterior mesoderm for development of the notochord [14]. A microarray analysis of gene expression showed that axial chordomas express high levels of brachyury, and this was supported by immunohistochemical studies demonstrating brachyury as a sensitive and specific marker for chordomas $[14,15]$. Treatment of these tumors is based on radical surgery combined with radiation therapy. Although treated aggressively, these tumours have a high degree of local recurrence [3].

Other lesions that can be confused with BNCT are metastatic carcinomas, particularly originating from clear cell carcinoma of the kidney $[1,10]$.

Considering their common origin as well as histological, immunohistochemical and ultrastructural similarities, BNCTs are thought to be the benign counterpart to chordomas $[3,10,11]$. It is not known however, whether BNCTs can be a chordoma precursors [1,11]. One report from 2002, do describe a chordoma observed adjacent to a benign notochordal lesion in the coccyx occurring concomitant with two BNCTs in the sacrum [1]. In 2005, a report was published describing two cases with BNCTs associated with miniature chordomas (incipient chordomas) [16]. Finally, one study (2007) identified BNCTs in 
7.3\% (6 of 82) of sacral/coccygeal resections performed for chordoma [11]. Recently, Kikuchi et al. reported two cases of pulmonary tumor with notochordal differentiation, suggestive of benign notochordal cell tumor of extraosseous origin [14]. These two cases suggest a new explanation for the histogenesis of extra-axial chordomas, indeed BNCTs may be a precursor lesion of not only conventional axial chordoma but also of extra-axial chordoma [14]. It is therefore important to consider the possible evolution of BNCT into chordomas.

\section{Conclusion}

The intraosseous benign notochordal cell tumor (BNCT) is a benign lesion from notochordal origin. Important histopathological observations, such as absence of cytonuclear atypia, mitosis or myxoid background, may help to distinguish BNCTs from the malignant chordoma. In addition, the clinical presentation and radiological imaging are important contributors to the correct diagnosis. To distinguish BNCTs from chordoma is important for treatment decisions and in predicting outcome, as the indolent nature of BNCTs only requires surveillance and has a good prognosis. Malignant transformation to chordomas is an undecided possibility.

\section{REFERENCES}

[1] H. Amer and M. Hameed, "Intraosseous Benign Notochordal Cell Tumor," Archives of Pathology \& Laboratory Medicine, Vol. 134, No. 2, 2010, pp. 283-288.

[2] W. L. Wang, J. H. Abramson, A. Ganguly and A. E. Rosenberg, "The Surgical Pathology of Notochordal Remnants in Adult Intervertebral Disks: A Report of 3 Cases,” American Journal of Surgical Pathology, Vol. 32, No. 8, 2008, pp. 1123-1129. http://dx.doi.org/10.1097/PAS.0b013e3181757954

[3] A. Chauvel, F. Taillat, O. Gille, et al., "Giant Vertebral Notochordal Rest: A Newentity Distinct from Chordoma," Histopathology, Vol. 47, No. 6, 2005, pp. 646-649. http://dx.doi.org/10.1111/j.1365-2559.2005.02168.x

[4] F. Mehnert, R. Beschorner, W. Küker, U. Hahn and T. Nägele, "Retroclival Ecchordosisphysaliphora: MR Imaging and Review of the Literature," American Journal of Neuroradiology, Vol. 25, No. 10, 2004, pp. 1851-1855.

[5] H. Luschka, "Die Altersveräenderungen der Zwischenwirbelknorpel," Virchows Archiv A, Pathological Anatomy and Histology, Vol. 9, No. 3, 1856, pp. 311-327. http://dx.doi.org/10.1007/BF01879395

[6] R. Virchow, "Untersuchungen ueber die Entwicklung des Schaedelgrundes,” G. Rimer, Berlin, 1857, p. 47.

[7] H. Ribbert and H. Steiner, "Uber die Ecchondrosis Phy- salifora Sphenooccipitalis,” Zentralbl Allg Pathol Anat, Vol. 5, 1894, pp. 457-461.

[8] T. Yamaguchi, S. Suzuki, H. Ishiiwa and Y. Ueda, "Intraosseous Benign Notochordalcell Tumours: Overlooked Precursors of Classic Chordomas?” Histopathology, Vol. 44, No. 6, 2004, pp. 597-602.

http://dx.doi.org/10.1111/j.1365-2559.2004.01877.x

[9] T. Yamaguchi, S. Suzuki, H. Ishiiwa, K. Shimizu and Y. Ueda, "Benign Notochordalcell Tumors: A Comparative Histological Study of Benign Notochordal Cell Tumors, Classic Chordomas, and Notochordal Vestiges of Fetal Intervertebral Disks,” American Journal of Surgical Pathology, Vol. 28, No. 6, 2004, pp. 756-761.

http://dx.doi.org/10.1097/01.pas.0000126058.18669.5d

[10] T. Yamaguchi, J. Iwata, S. Sugihara, et al., "Distinguishing Benign Notochordalcell Tumors from Vertebral Chordoma,” Skeletal Radiology, Vol. 37, No. 4, 2008, pp. 291-299. http://dx.doi.org/10.1007/s00256-007-0435-y

[11] V. Deshpande, G. P. Nielsen, D. I. Rosenthal and A. E. Rosenberg, "Intraosseous Benign Notochord Cell Tumors (BNCT): Further Evidence Supporting a Relationship Tochordoma,” American Journal of Surgical Pathology, Vol. 31, No. 10, 2007, pp. 1573-1577. http://dx.doi.org/10.1097/PAS.0b013e31805c9967

[12] T. Nishiguchi, K. Mochizuki, M. Ohsawa, T. Inoue, K. Kageyama, A. Suzuki, T. Takami and Y. Miki, "Differentiating Benign Notochordal Cell Tumors from Chordomas: Radiographic Features on MRI, CT, and Tomography,” American Journal of Roentgenology, Vol. 196, No. 3, 2011, pp. 644-650. http://dx.doi.org/10.2214/AJR.10.4460

[13] S. H. Ng, S. F. Ko, Y. L. Wan, L. M. Tang and Y. S. Ho, "Cervical Ecchordosis Physaliphora: CT and MR Features,” British Journal of Radiology, Vol. 71, No. 843, 1998, pp. 329-331.

[14] Y. Kikuchi, T. Yamaguchi, H. Kishi, K. Azuhata, G. Kimizuka, K. Hiroshima and Y. Nakatani, "Pulmonary Tumor with Notochordal Differentiation: Report of Two Cases Suggestive of Benign Notochordal Cell Tumor of Extraosseous Origin," American Journal of Surgical Pathology, Vol. 35, No. 8, 2011, pp. 1158-1164. http://dx.doi.org/10.1097/PAS.0b013e318220e085

[15] R. Tirabosco, D. C. Mangham, A. E. Rosenberg, et al., "Brachyury Expression in Extra-Axial Skeletal and Soft Tissue Chordomas: A Marker That Distinguishes Chordoma from Mixed Tumor/Myoepithelioma/Parachordoma in Soft Tissue,” American Journal of Surgical Pathology, Vol. 32, No. 4, 2008, pp. 572-580. http://dx.doi.org/10.1097/PAS.0b013e31815b693a

[16] T. Yamaguchi, H. Watanabe-Ishiiwa, S. Suzuki, Y. Igarashi and Y. Ueda, "Incipientchordoma: A Report of Two Cases of Early-Stage Chordoma Arising from Benign Notochordal Cell Tumors,” Modern Pathology, Vol. 18, No. 7, 2005, pp. 1005-1010. http://dx.doi.org/10.1038/modpathol.3800378 